\title{
The Area Under the Disease Progress Stairs: Calculation, Advantage, and Application
}

\author{
Ivan Simko and Hans-Peter Piepho
}

First author: U.S. Department of Agriculture-Agricultural Research Service, Crop Improvement and Protection Research Unit, 1636 East Alisal Street, Salinas, CA 93905; and second author: Institut für Kulturpflanzenwissenschaften, Universität Hohenheim, Fruwirthstrasse 23, D-70593 Stuttgart, Germany.

Accepted for publication 16 November 2011.

\begin{abstract}
Simko, I., and Piepho, H.-P. 2012. The area under the disease progress stairs: Calculation, advantage, and application. Phytopathology 102:381389.

The area under the disease progress curve (AUDPC) is frequently used to combine multiple observations of disease progress into a single value. However, our analysis shows that this approach severely underestimates the effect of the first and last observation. To get a better estimate of

the disease progress stairs (AUDPS). The AUDPS approach improves the estimation of disease progress by giving a weight closer to optimal to the first and last observations. Analysis of real data indicates that AUDPS outperforms AUDPC in most of the tested trials and may be less precise than AUDPC only when assessments in the first or last observations have a comparatively large variance. We propose using AUDPS and its standardized (sAUDPS) and relative (rAUDPS) forms when combining multiple observations from disease progress experiments into a single value.
\end{abstract} disease progress, we have developed a new formula termed the area under
Two types of resistance to pathogens have been described and used in plant breeding. One type is a qualitative resistance that is conferred by a series of major dominant genes that render the host incompatible with the pathogen. The other type is a quantitative resistance that is usually conferred by the effect of multiple genes of minor effects $(1,9)$. In quantitative resistance, where differences in level of resistance are usually less distinct, measuring disease progress is important for understanding plant-pathogen interaction. Disease typically starts at a low level, gradually increasing in incidence and/or severity over time. Progress of disease on plants is usually observed several times during pathogen epidemics. Extent of disease is assessed at each observation using scales that are based on disease incidence, severity, or a combination of both. To combine these repeated observations into a single value, Van der Plank (chapter 12 of literature citation 13) proposed calculating the area under the disease progress curve (AUDPC). If progress of disease fits one of the characterized models $(3,4)$, parameters estimated from this model can be used for calculating AUDPC. However, if there is no credible evidence that one of the standard models fits the observed disease progress data or if the disease progress data varies substantially in shape for different factor levels, then the empirical determination of AUDPC is necessary (5).

Our analysis shows that the AUDPC approach systematically undervalues the effects of the first and last observations. The main objectives of this study were (i) to present a new method for combining disease progress data that would give an appropriate weight to these two observations, and (ii) to compare the performance of this new method with AUDPC.

Corresponding author: I. Simko; E-mail address: ivan.simko@ars.usda.gov

* The $\boldsymbol{e}$-Xtra logo stands for "electronic extra" and indicates that the online version contains two supplemental tables and one supplemental material.

http://dx.doi.org/10.1094/PHYTO-07-11-0216

This article is in the public domain and not copyrightable. It may be freely reprinted with customary crediting of the source. The American Phytopathological Society, 2012.

\section{THEORY AND APPROACHES}

Formulas for AUDPC and AUDPS. A common approach to determine AUDPC is through a simple midpoint (trapezoidal) rule (Fig. 1A) that breaks up a disease progress curve into a series of trapezoids, calculating the area of each, and then adding up the areas (5):

$$
A U D P C=\sum_{i=1}^{n-1} \frac{y_{i}+y_{i+1}}{2} \times\left(t_{i+1}-t_{i}\right)
$$

where $y_{i}$ is an assessment of a disease (percentage, proportion, ordinal score, etc.) at the $i$ th observation, $t_{i}$ is time (in days, hours, etc.) at the $i$ th observation, and $n$ is the total number of observations. Rearrangement of this formula shows that AUDPC is calculated by multiplying each assessment value by its respective weight (Fig. 1B)

$$
A U D P C=\left[y_{1} \times \frac{t_{2}-t_{1}}{2}\right]+\left[\sum_{i=2}^{n-1}\left(y_{i} \times \frac{t_{i+1}-t_{i-1}}{2}\right)\right]+\left[y_{n} \times \frac{t_{n}-t_{n-1}}{2}\right]
$$

where $y_{1}$ and $y_{n}$ are assessments at the first and last observations, respectively, and $t_{1}, t_{2}, t_{n-1}$, and $t_{n}$ are the times of the first, second, penultimate, and last observations.

The weight for each assessment is the duration between the midpoint of the previous time interval to the midpoint of the subsequent time interval

$$
\left(v_{i}=\frac{t_{i+1}-t_{i-1}}{2}=\frac{t_{i+1}-t_{i}}{2}+\frac{t_{i}-t_{i-1}}{2}\right)
$$

Thus, all weights are calculated by extrapolating in two directions, with the exception of the first and the last assessments for which weight is extrapolated in one direction only $\left[v_{1}=\left(t_{2}-t_{1}\right) / 2\right.$ for $y_{1}$, and $v_{n}=\left(t_{n}-t_{n-1}\right) / 2$ for $\left.y_{n}\right]$. For example, if observations are performed every tenth day, then each assessment is multiplied by the weight of $10: 10 / 2+10 / 2=10$, with the exception of the first and last assessments that are multiplied only by the weight of five: $10 / 2=5$. This leads to a substantial undervaluation of the first and the last assessments in comparison to all other assessments. It is possible that in some 
special cases this lower relative weight may be desirable, but for the majority of evaluations there is no added benefit in giving only half a weight to assessments at these two observations. To give the appropriate importance to the first and the last assessments, weights for these assessments should also be extrapolated in both directions. We suggest extrapolating a weight in the missing direction using half of the average interval duration between observations $(D /[2 \times(n-1)])$, where $D=t_{n}-t_{1}$. This new method that is used to calculate total area (including adjusted weight for the first and the last assessments) is termed area under the disease progress stairs (AUDPS) (Fig. 1C). The term "stairs" was chosen to reflect the fact that AUDPS corresponds to the area under the step function shown in Figure 1C, but not to the area under a curve or polygon obtained by connecting dots representing the assessed values for individual observations (Fig. 1A and $\mathrm{B}$ ). The mathematical formula for AUDPS is

$$
\begin{aligned}
& A U D P S=\left[y_{1} \times\left\{\frac{t_{2}-t_{1}}{2}+\frac{D}{2(n-1)}\right\}\right]+\left[\sum_{i=2}^{n-1}\left(y_{i} \times \frac{t_{i+1}-t_{i-1}}{2}\right)\right]+\left[y_{n} \times\left\{\frac{t_{n}-t_{n-1}}{2}+\frac{D}{2(n-1)}\right\}\right] \\
& =\left[\sum_{i=1}^{n-1} \frac{y_{i}+y_{i+1}}{2} \times\left(t_{i+1}-t_{i}\right)\right]+\left[\frac{y_{1}+y_{n}}{2} \times \frac{D}{n-1}\right]=A U D P C+\left[\frac{y_{1}+y_{n}}{2} \times \frac{D}{n-1}\right]
\end{aligned}
$$
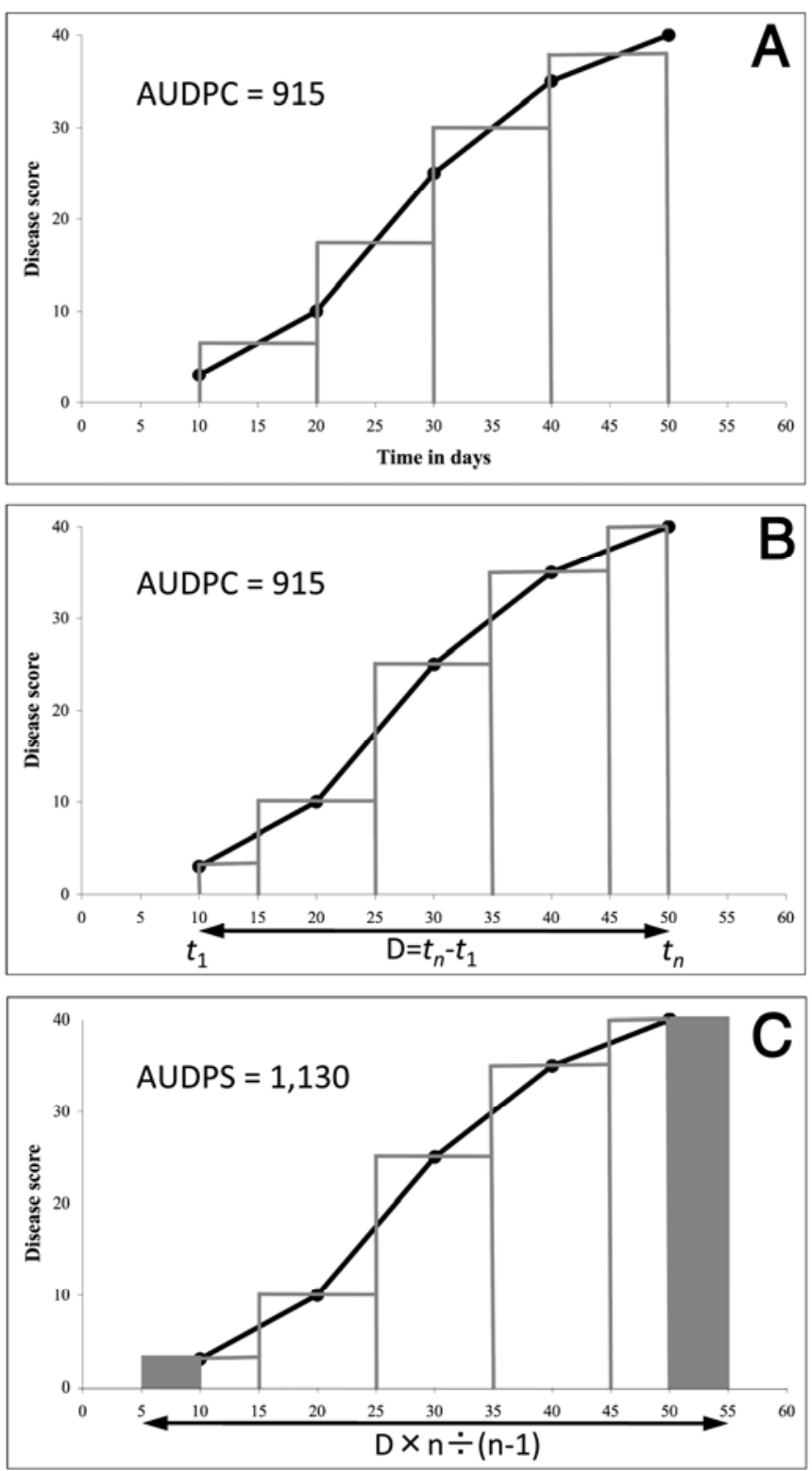

Fig. 1. Graphical interpretation of area under the disease progress curve (AUDPC) and area under the disease progress stairs (AUDPS) calculations. Black line indicates a disease progress curve. A, Gray line indicates rectangles that are used to calculate AUDPC based on the trapezoidal rule. B, Rearrangement of the formula shows that the same AUDPC can be calculated by multiplying each assessment value by its weight. In this example each value is multiplied by 10, with the exception of the first and last assessments that are multiplied by 5. C, Calculation of AUDPS. Gray bars show the area that is added to AUDPC after adjusting weights for the first and last observations.
When all observations are performed at regular intervals, the combined weight of all assessments is equal to $D n /(n-1)$ and the AUDPS formula becomes

$$
A U D P S=\bar{y} \times \frac{D n}{n-1}
$$

where $\bar{y}$ is the arithmetic mean of all assessments. When observations are performed at every time unit (e.g., hourly, daily, weekly, etc.), $D=n-1$ and the formula can be simplified as

$$
A U D P S=\bar{y} \times n
$$

Standardization of AUDPC and AUDPS and their statistical properties. AUDPC and AUDPS cover different time spans. While the time span for AUDPC is $D$, for AUDPS it is $D n /(n-1)$. To make the two measures more directly comparable, both should be standardized. A standardized version of AUDPC, denoted here as sAUDPC (5), can be written as

$$
s A U D P C=\frac{A U D P C}{D}
$$

In the same way a standardized form of AUDPS is written as

$$
s A U D P S=\frac{A U D P S \times(n-1)}{D n}
$$

The standardized versions of AUDPC and AUDPS have the general form

$$
L=\sum_{i=1}^{n} w_{i} y_{i}
$$

where $w_{i}$ indicates weights, subject to the constraint

$$
\sum_{i=1}^{n} w_{i}=1
$$

and $y_{i}$ indicates assessments at observations $i=1, \ldots, n$. For example, with AUDPC we have

$$
w_{i}=v_{i} / \sum_{j=1}^{n} v_{j}=v_{i} / D
$$

where $v_{1}=\left(t_{2}-t_{1}\right) / 2, \quad v_{i}=\left(t_{i+1}-t_{i-1}\right) / 2$ for $i=2, \ldots, n-1$, and $v_{n}=\left(t_{n}-t_{n-1}\right) / 2$. The measures sAUDPC and sAUDPS differ in the definition of weights. The definition of weights follows slightly different concepts of measuring disease progress, and the choice of measure should be primarily based on a choice of concept. However, it is also useful to study the statistical properties of alternative measures. One possible approach is to ask what choice of weights maximizes the precision.

With $w=\left(w_{1}, w_{2}, . ., w_{n}\right)^{T}$ and $y=\left(y_{1}, y_{2}, . ., y_{n}\right)^{T}$, where the superscripted $T$ denotes a transpose of a matrix or vector, our measures can be written as $L=w^{T} y$. This has a variance of $\operatorname{var}(L)=w^{T} V w$, 
where $V$ is the variance-covariance matrix of $y$. The optimal choice of $w$ will minimize $\operatorname{var}(L)$. If we impose the constraint

$$
\sum_{i=1}^{n} w_{i}=1
$$

then using the method of Lagrange multipliers, the optimal weights are found to be

$$
w=\left(w_{1}, w_{2}, \ldots, w_{n}\right)^{T}=\left(1^{T} V^{-1} 1\right)^{-1} 1^{T} V^{-1}
$$

where 1 is a vector of ones. Some of these optimal weights may become negative, depending on the form of $V$. We may impose the further constraint that $w_{i} \geq 0$ for all $i$. In this case, there is no easily derived simple expression for the optimal weights, but we may use numerical optimization routines such as the NLPTR function in SAS/IML 9.2 (SAS Institute, Cary, NC). A theoretical investigation of optimal weights for special forms of $V$ is given in Appendix A.

To further study the properties of AUDPC and AUDPS, we modified weights of the first and last observation to minimize the overall variance of $L$ for empirical data sets. In other words, we assigned the same relative weights as in AUDPC and AUDPS to all observations from 2 to $n-1$, and then tested weights for the first and last observation to achieve a minimum variance. These optimal weights were determined by the same method as described above, using triplet $y_{1}, y_{n}$, and a weighted mean of $y_{2}$ to $y_{n-1}$ computed as

$$
m=\frac{\sum_{i=2}^{n-1} v_{i} y_{i}}{\sum_{i=2}^{n-2} v_{i}}, \text { where } v_{i}=\left(t_{i+1}-t_{i-1}\right) / 2 \text { for } i=2, \ldots, n-1 .
$$

We then optimized weights $w_{1}, c$, and $w_{n}$ in

$$
L=w_{1} y_{1}+c m+w_{n} y_{n}
$$

subject to the constraints $w_{1}+c+w_{n}=1$ and $w_{1}, c, w_{n} \geq 0$. Note that weights for intermediate observations are

$$
w_{i}=\frac{c v_{i}}{\sum_{i=2}^{n-2} v_{i}}
$$

for $i=2, \ldots, n-1$. Further note that when all $v_{i}$ are the same, we have $w_{i}=c /(n-2)$. A justification of why we use the weighted mean $m$ in equation 10 is given in Appendix B.

Tests of performance. Performance of AUDPC/sAUDPC and AUDPS/sAUDPS formulas was evaluated on a set of 50 trials from different plant-pathogen (or plant-fungicide interaction) systems that are illustrative both of monocyclic and polycyclic diseases (Supplemental Table 1). The LB trials $(1,8)$ represent interaction of potato (Solanum tuberosum L. and Solanum berthaultii L.) with Phytophthora infestans (Mont.) de Bary, while DM, PL, LD, Di, SL, and Tr trials represent interaction of lettuce (Lactuca sativa L. and Lactuca serriola L.) with Bremia lactucae Regel (DM), Erysiphe cichoracearum DC ex Mérat (PL), Sclerotinia minor Jaggar (LD), Tomato bushy stunt virus and Lettuce necrotic stunt virus (Di) $(11,12)$, a combination of biotic and abiotic factors causing salad deterioration (SL), and Triforinebased fungicides (Tr) (10). The CS, PS, and PM data originate from trials testing resistance of sugar beet (Beta vulgaris L.) to Cercospora beticola Sacc. (CS), Erysiphe polygoni DC. (syn. E. betae (Vanha) Weltzien) (PS), and melon (Cucumis melo L.) to Podosphaera xanthii (Castagne) Braun \& Shishkoff (PM). To find out which of the two methods performs better, we compared a number of different statistics. The $F$ values, root of mean square error (RMSE), and coefficient of variation (CV) were obtained from one-way analysis of variance (ANOVA) calculated with JMP 6.0.3 (SAS Institute). Optimal weights were estimated as de- scribed above using the nonlinear optimization subroutine NLPTR in the IML procedure of SAS 9.2 (SAS Institute). The variancecovariance matrix $V$ was estimated by the MIXED procedure specifying an unstructured model. The Euclidean distance (ED) between relative optimal weights and relative actual weights used for calculation of AUDPC and AUDPS was calculated as

$$
E D(o, a)=\sqrt{\left(w_{1}^{(o)}-w_{1}^{(a)}\right)^{2}+\left(c^{(o)}-c^{(a)}\right)^{2}+\left(w_{n}^{(o)}-w_{n}^{(a)}\right)^{2}}
$$

where superscripts (o) and (a) indicate optimal and actual weights, respectively.

Statistics used to test performance. To compare performance of AUDPC/sAUDPC and AUDPS/sAUDPS methods, we used $F$ value, RMSE, and CV from ANOVA. However, some of these statistics might have noticeable weaknesses. For example, if either AUDPC or AUDPS overestimates the difference between two accessions because of the shape of the disease progress curve, a higher $F$ value will result from bias in the estimate, rather than improved precision. $\mathrm{CV}$ avoids this kind of bias because it does not depend on differences between accessions. However, CV is not invariant to shift transformations (addition of a constant). The RMSE of standardized values is probably the most suitable of the three statistics for our evaluations because it does not depend on differences between accessions and is unaffected if some shiftscale transformation is applied to sAUDPC or sAUDPS. Nevertheless, for comparison purposes we show all three values together with ED in Table 1. Relative performance of the two models (sAUDPC versus sAUDPS) was evaluated by comparing frequency of higher precision calculated for $F$ value, RMSE, CV, and ED from 50 trials. The significance of difference between two frequencies was tested using a two-sample paired sign test.

\section{RESULTS}

Trials used for testing of the two formulas represent a range of different plant-pathogen systems and evaluation methods. The number of tested accessions range from 3 to 479 , the number of replications within a trial is (on average) from less than 2 to 12 , the duration from the first to last observation is from 3 to 120 days, the number of observations is from 3 to 18 , average length of interval is from 1 to 16.5 days, and evaluations are based on six different rating scales. There may be some occasional heterogeneity of variance between accessions at specific time points because of differences in pathogen infection levels. This was ignored in ANOVA and optimal weight calculations because we could not find a simple approach to account for heteroscedasticity in the same way for all data sets. While some improvement would be possible by more sophisticated modeling, we believe that for the purpose of the present analyses it is satisfactory to work with the assumption of the constant variance.

Results from one-way ANOVA are summarized in Table 1. Comparison of $F$ values indicates that sAUDPS outperforms sAUDPC in 35 of 50 trials. RMSE is smaller for sAUDPS in 46 trials; while 42 trials have smaller CV when sAUDPS was used for combining data from disease progress observations.

Theoretical optimal weights were calculated for the first and last observations, and the combination of all other 'central' observations. The optimal weights were based on the assumption that smaller overall variance is favorable when assessments from all observations are combined into a single value. For comparison across data sets, it may be preferable to use relative weights. The actual relative weights used by AUDPC and AUDPS can be computed as follows:

$$
w_{i}=\frac{v_{i}}{\sum_{i=1}^{n} v_{i}} \text { and } c=\frac{\sum_{i=2}^{n-1} v_{i}}{\sum_{i=1}^{n} v_{i}}
$$


When weights used for calculation of sAUDPC and sAUDPS were compared with the theoretical optimal weights (Supplemental Table 2), sAUDPS outperformed sAUDPC in 41 trials for weight for the first observation $\left(w_{1}\right)$ and in 27 trials for weight for the last observation $\left(w_{n}\right)$. Based on their similarity to the optimal weights, 50 trials can be categorized into four groups: a group of 18 trials that have real weights of sAUDPS closer to the optimal weights for $w_{1}, c$, and $w_{n}$ (Fig. 2, example CS5); a group of nine trials that have real weights of sAUDPS closer to the optimal weights for $c$ and $w_{n}$ (Fig. 2, example Di); a group of 22 trials that have real weights of sAUDPS closer to the optimal weight for $w_{1}$ and $c$ (Fig. 2, example LD4); and a single trial that have real weights of sAUDPS closer to the optimal weight for $w_{1}$ only (Fig. 2, example PL1). When the Euclidean metric was used to calculate the overall distance between optimal and actual weights, sAUDPS outperformed sAUDPC in 49 trials, the single exception being the trial PL1 (Table 1).

Each of the four metrics ( $F$ value, RMSE, CV, and ED) showed a statistically significant frequency of higher precision for sAUDPS based on a sign test with $P$ values ranging from 0.0066 to less than 0.0001 (Table 1). From 50 analyzed trials, PL1 is the only one in which all four statistics indicate that sAUDPC outperforms sAUDPS. In contrast, all four statistics imply that sAUDPS outperforms sAUDPC in 34 of 50 trials.

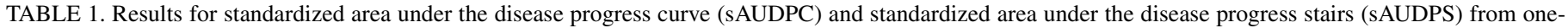
way analysis of variance and Euclidean distances to optimal weights ${ }^{\mathrm{a}}$

\begin{tabular}{|c|c|c|c|c|c|c|c|c|c|c|c|}
\hline Trial & Formula & $F$ value & $\mathrm{RMSE}^{\mathrm{b}}$ & $\mathrm{CV}^{\mathrm{c}}$ & $\mathrm{ED}^{\mathrm{d}}$ & Trial & Formula & $F$ value & $\mathrm{RMSE}^{\mathrm{b}}$ & $\mathrm{CV}^{\mathrm{c}}$ & $\mathrm{ED}^{\mathrm{d}}$ \\
\hline \multirow[t]{2}{*}{ LB1 } & sAUDPC & 32.514 & 3.344 & 0.751 & 1.278 & \multirow[t]{2}{*}{ LD7 } & sAUDPC & 2.551 & 17.068 & 28.460 & 0.791 \\
\hline & sAUDPS & 30.553 & 3.378 & 0.724 & 1.178 & & sAUDPS & 2.573 & 16.200 & 28.254 & 0.596 \\
\hline \multirow[t]{2}{*}{ LB2 } & sAUDPC & 3.017 & 15.569 & 0.463 & 1.019 & \multirow[t]{2}{*}{ LD8 } & sAUDPC & 7.888 & 8.706 & 16.788 & 1.288 \\
\hline & sAUDPS & 3.136 & 14.082 & 0.422 & 0.831 & & sAUDPS & 9.041 & 8.174 & 16.033 & 1.189 \\
\hline \multirow[t]{2}{*}{ LB3 } & sAUDPC & 21.070 & 5.808 & 0.376 & 1.323 & \multirow[t]{2}{*}{ LD9 } & sAUDPC & 79.219 & 14.610 & 40.260 & 1.000 \\
\hline & sAUDPS & 22.646 & 5.638 & 0.353 & 1.239 & & sAUDPS & 80.234 & 14.511 & 40.269 & 0.866 \\
\hline \multirow[t]{2}{*}{ LB4 } & sAUDPC & 3.936 & 13.065 & 21.422 & 1.070 & \multirow[t]{2}{*}{ LD10 } & sAUDPC & 38.131 & 15.207 & 40.614 & 0.613 \\
\hline & sAUDPS & 3.884 & 12.624 & 20.763 & 0.895 & & sAUDPS & 37.241 & 14.969 & 41.049 & 0.408 \\
\hline \multirow[t]{2}{*}{ LB5 } & sAUDPC & 1.506 & 9.098 & 22.530 & 1.092 & \multirow[t]{2}{*}{ LD11 } & sAUDPC & 7.892 & 18.045 & 83.822 & 0.966 \\
\hline & sAUDPS & 1.518 & 7.510 & 17.810 & 0.943 & & sAUDPS & 7.861 & 17.767 & 83.679 & 0.839 \\
\hline \multirow[t]{2}{*}{ LB6 } & sAUDPC & 2.993 & 8.357 & 16.709 & 1.123 & \multirow[t]{2}{*}{ LD12 } & sAUDPC & 0.702 & 12.609 & 147.546 & 1.027 \\
\hline & sAUDPS & 3.309 & 6.800 & 13.665 & 0.993 & & sAUDPS & 0.700 & 12.084 & 147.975 & 0.885 \\
\hline \multirow[t]{2}{*}{ LB7 } & sAUDPC & 9.494 & 4.233 & 8.407 & 0.950 & \multirow[t]{2}{*}{ LD13 } & sAUDPC & 2.717 & 15.153 & 51.514 & 0.787 \\
\hline & sAUDPS & 11.717 & 3.564 & 7.086 & 0.792 & & sAUDPS & 2.741 & 14.645 & 50.595 & 0.602 \\
\hline \multirow[t]{2}{*}{ LB8 } & sAUDPC & 4.193 & 8.547 & 14.174 & 1.059 & \multirow[t]{2}{*}{ LD14 } & sAUDPC & 0.608 & 22.231 & 71.241 & 0.966 \\
\hline & sAUDPS & 4.921 & 6.943 & 11.962 & 0.918 & & sAUDPS & 0.576 & 20.252 & 69.506 & 0.839 \\
\hline \multirow[t]{2}{*}{ LB9 } & sAUDPC & 1.399 & 7.801 & 21.523 & 1.066 & LD15 & sAUDPC & 2.626 & 19.189 & 38.392 & 0.911 \\
\hline & sAUDPS & 1.475 & 6.952 & 17.535 & 0.913 & & sAUDPS & 2.508 & 19.122 & 39.144 & 0.788 \\
\hline SL1 & sAUDPC & 15.262 & 1.008 & 0.154 & 1.185 & LD16 & sAUDPC & 4.144 & 9.995 & 21.496 & 0.938 \\
\hline & sAUDPS & 17.712 & 0.912 & 0.143 & 1.071 & & sAUDPS & 4.320 & 9.355 & 20.531 & 0.767 \\
\hline SL2 & sAUDPC & 12.365 & 0.903 & 0.118 & 1.318 & Di & sAUDPC & 21.143 & 8.909 & 0.345 & 0.493 \\
\hline & sAUDPS & 13.576 & 0.843 & 0.112 & 1.240 & & sAUDPS & 24.763 & 8.189 & 0.317 & 0.337 \\
\hline SL3 & sAUDPC & 54.128 & 0.572 & 0.067 & 1.327 & $\operatorname{Tr} 1$ & sAUDPC & 243.269 & 4.685 & 10.877 & 1.330 \\
\hline & sAUDPS & 55.419 & 0.551 & 0.066 & 1.254 & & sAUDPS & 233.352 & 4.692 & 11.062 & 1.271 \\
\hline SL4 & sAUDPC & 17.094 & 0.669 & 0.088 & 1.318 & $\operatorname{Tr} 2$ & sAUDPC & 20.650 & 8.811 & 15.007 & 1.004 \\
\hline & sAUDPS & 16.939 & 0.621 & 0.084 & 1.241 & & sAUDPS & 21.344 & 8.159 & 14.077 & 0.862 \\
\hline SL5 & sAUDPC & 20.222 & 1.366 & 18.469 & 1.102 & PL1 & sAUDPC & 8.381 & 0.569 & 29.116 & 0.306 \\
\hline & sAUDPS & 21.177 & 1.229 & 17.084 & 0.968 & & sAUDPS & 8.039 & 0.599 & 30.170 & 0.445 \\
\hline SL6 & sAUDPC & 2.393 & 1.027 & 15.144 & 1.352 & PL2 & sAUDPC & 8.426 & 0.328 & 14.063 & 1.137 \\
\hline & sAUDPS & 2.403 & 0.971 & 14.512 & 1.298 & & sAUDPS & 13.965 & 0.251 & 10.658 & 0.996 \\
\hline SL7 & sAUDPC & 9.920 & 0.849 & 9.783 & 1.319 & CS1 & sAUDPC & 5.569 & 1.459 & 41.387 & 0.346 \\
\hline & sAUDPS & 10.299 & 0.825 & 9.663 & 1.242 & & sAUDPS & 5.305 & 1.448 & 40.585 & 0.184 \\
\hline SL8 & sAUDPC & 13.656 & 0.737 & 9.883 & 1.242 & $\mathrm{CS} 2$ & sAUDPC & 18.492 & 0.457 & 6.312 & 0.176 \\
\hline & sAUDPS & 13.664 & 0.670 & 9.797 & 1.154 & & sAUDPS & 18.154 & 0.455 & 6.244 & 0.176 \\
\hline SL9 & sAUDPC & 8.677 & 0.843 & 18.361 & 1.075 & $\mathrm{CS} 3$ & sAUDPC & 11.915 & 0.481 & 13.158 & 0.823 \\
\hline & sAUDPS & 8.605 & 0.700 & 15.040 & 0.923 & & sAUDPS & 12.190 & 0.460 & 12.705 & 0.656 \\
\hline SL10 & sAUDPC & 19.576 & 0.779 & 15.254 & 0.928 & CS4 & sAUDPC & 1.610 & 0.532 & 23.672 & 0.646 \\
\hline & sAUDPS & 20.689 & 0.652 & 12.811 & 0.746 & & sAUDPS & 1.611 & 0.514 & 22.647 & 0.458 \\
\hline DM & sAUDPC & 6.496 & 0.461 & 0.251 & 0.838 & CS5 & sAUDPC & 2.567 & 0.519 & 12.177 & 0.727 \\
\hline & sAUDPS & 6.602 & 0.415 & 0.230 & 0.641 & & sAUDPS & 2.598 & 0.481 & 11.644 & 0.552 \\
\hline LD1 & sAUDPC & 9.286 & 11.865 & 0.291 & 0.795 & PS1 & sAUDPC & 14.048 & 0.506 & 68.901 & 0.713 \\
\hline & sAUDPS & 9.540 & $\mathbf{1 1 . 5 5 7}$ & 0.281 & 0.617 & & sAUDPS & 14.679 & 0.502 & 64.886 & 0.609 \\
\hline LD2 & sAUDPC & 2.035 & 10.176 & 44.290 & 1.196 & PS2 & sAUDPC & 5.033 & 0.332 & $\mathbf{1 7 7 . 9 1 7}$ & 1.010 \\
\hline & sAUDPS & 2.127 & 9.610 & 41.580 & 1.053 & & sAUDPS & 4.573 & 0.379 & 179.374 & 0.873 \\
\hline LD3 & sAUDPC & 2.812 & 20.020 & 50.078 & 1.014 & PS3 & sAUDPC & 58.732 & 0.625 & 15.812 & 0.428 \\
\hline & sAUDPS & 2.981 & 19.600 & 49.186 & 0.800 & & sAUDPS & 60.302 & 0.597 & 15.537 & 0.234 \\
\hline LD4 & sAUDPC & 1.132 & 17.855 & 32.144 & 0.602 & PM & sAUDPC & 34.609 & 0.933 & 14.930 & 1.071 \\
\hline & sAUDPS & 1.109 & 17.574 & 32.166 & 0.435 & & sAUDPS & 42.074 & 0.744 & 13.296 & 0.917 \\
\hline LD5 & sAUDPC & 56.028 & 8.395 & 18.308 & 0.899 & Total $^{\mathrm{e}}$ & sAUDPC & 15 & 4 & 8 & 1 \\
\hline & sAUDPS & 61.869 & 7.453 & 16.738 & 0.736 & & sAUDPS & 35 & 46 & 42 & 49 \\
\hline LD6 & sAUDPC & 5.675 & 8.773 & 47.374 & 1.168 & Sign test & $P$ value & 0.0066 & $<0.0001$ & $<0.0001$ & $<0.0001$ \\
\hline & sAUDPS & 6.228 & 8.504 & 45.347 & 1.026 & & & & & & \\
\hline
\end{tabular}

a Values for sAUDPC or sAUDPS in each trial that indicate higher precision (higher $F$ value, and lower RMSE, CV, and ED) are in bold type.

b $\mathrm{RMSE}=$ root of means square error.

c $\mathrm{CV}=$ coefficient of variation.

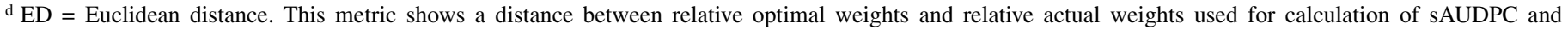
sAUDPS. The optimal and actual weights are shown in Supplemental material 1.

e Total number of sAUDPC and sAUDPS values with higher precision. The $P$ values are from a two-sample paired sign test. 


\section{DISCUSSION}

An alternative approach for determining optimal weights. Consideration of optimal weights leads to an alternative, selfweighting analysis. The idea is to estimate $V$ from the data and use it to determine optimal weights. Then compute

$$
L=\sum_{i=1}^{n} w_{i} y_{i}
$$

for each plot using optimal weights. It may be conjectured that this approach maximizes power to separate treatments. Estimation of $V$ can be done by a linear model for the repeated measures taken on the same plot (6). In estimating $V$, all effects except plot error can be taken as fixed. If $V$ were known, the resulting procedure would be exact. In practice, an estimator needs to be used. Provided the estimator is consistent and the number of plots is large, the procedure should be approximately valid. The smallsample behavior could be investigated by simulation.

A possible criticism of this procedure is that the definition of $L$ would be data-dependent and thus comparison across different studies would be difficult. Moreover, when combining different trials, optimal weights would differ between trials, meaning that in each trial a different definition of $L$ is used. Therefore, it may be better to use a fixed definition of $L$, as in AUDPC and AUDPS.

Alternative methods of extrapolating the weight in the missing direction. To extrapolate weight in a missing direction for the first and last observation we suggested using a half of the average interval duration between observations. There are, of course, other methods that could be used instead. For example, using the full length of the first interval as weight for the first observation and similarly using a length of the last interval as weight for the last observation. To choose the most appropriate weighting for practical application we analyzed 50 trials for which AUDPC was published (a list is available from authors). These data indicate that approximately $60 \%$ of trials use intervals with equal length. Another $20 \%$ of trials have intervals with a CV less than 0.2 . The remaining $20 \%$ of trials have a CV between 0.2 and 0.4 . Because majority of trials have a very low variability in the length of intervals, using the average interval duration appears to be a reasonable way of estimating missing weight in a majority of trials.

AUDPC versus AUDPS performance. Results that are summarized in Table 1 and Figure 2 indicate that most of the tested trials have more favorable values when AUDPS is applied to the disease progress data. To find out why all trails do not perform the same way, we plotted mean values and RMSE from individual observations (Fig. 3). These graphs show that sAUDPC outperforms sAUDPS in the trials where variance of assessments is still substantially growing at the last observation. This finding confirms our theoretical expectations that when assessments in the first or last observations have a comparatively large variance, sAUDPS may be less precise than sAUDPC. Our tests imply that a relative

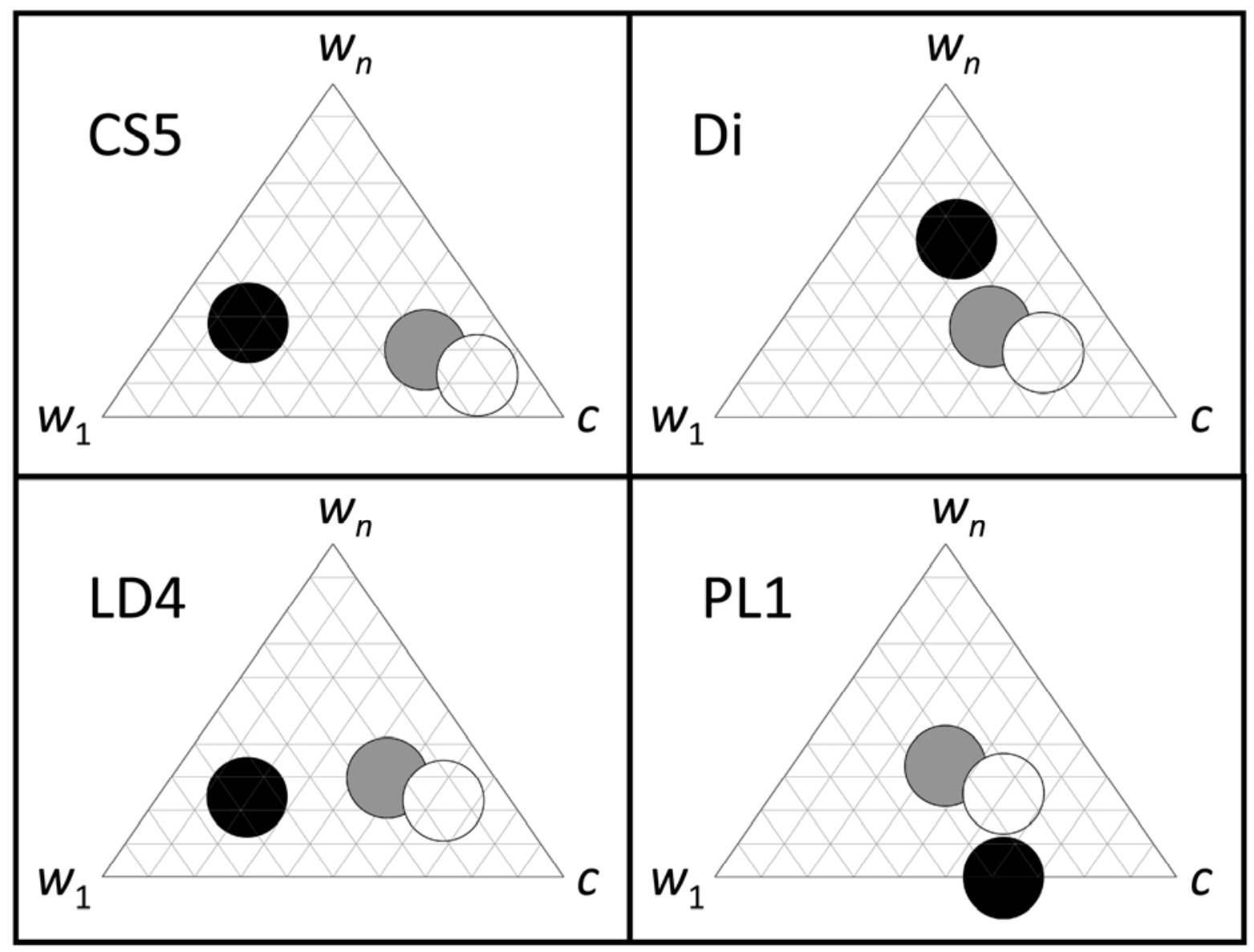

Fig. 2. Ternary plots of the relative optimal and relative real weights used for calculation of standardized area under the disease progress curve (sAUDPC) and standardized area under the disease progress stairs (sAUDPS). CS5 represents a group of 18 trials where real weights of sAUDPS are closer to the optimal weights for $w_{l}, c$, and $w_{n}$; Di represents a group of nine trials where real weights of sAUDPS are closer to the optimal weights for $c$ and $w_{n}$; LD4 represents a group of 22 trials where real weights of sAUDPS are closer to the optimal weights for $w_{1}$ and $c$; and PL1 is a single trial where real weights of sAUDPS are closer to the optimal weights for $w_{1}$ only. All trials with the exception of PL1 have the Euclidean distance between optimal and actual weights smaller for sAUDPS than for sAUDPC. Each side of the triangle represents a value of 0 , while the point of the triangle opposite to that side representing a value of 1 . Values of relative weights are indicated on the plot by circles of different color: black $=$ optimal, white $=$ sAUDPC, and gray $=$ sAUDPS. Values used for ternary plots are provided in Supplemental Table 2 . 
performance of the two methods is independent from the mean of assessments or the shape of disease progress curve, but it is affected by variance of assessments in individual observations and correlation between observations.
For a special case, we can theoretically predict when sAUDPS outperforms sAUDPC. Assuming equally spaced observations, and the perfect positive correlation between assessments in each accession, the relative performance of sAUDPC and sAUDPS de-

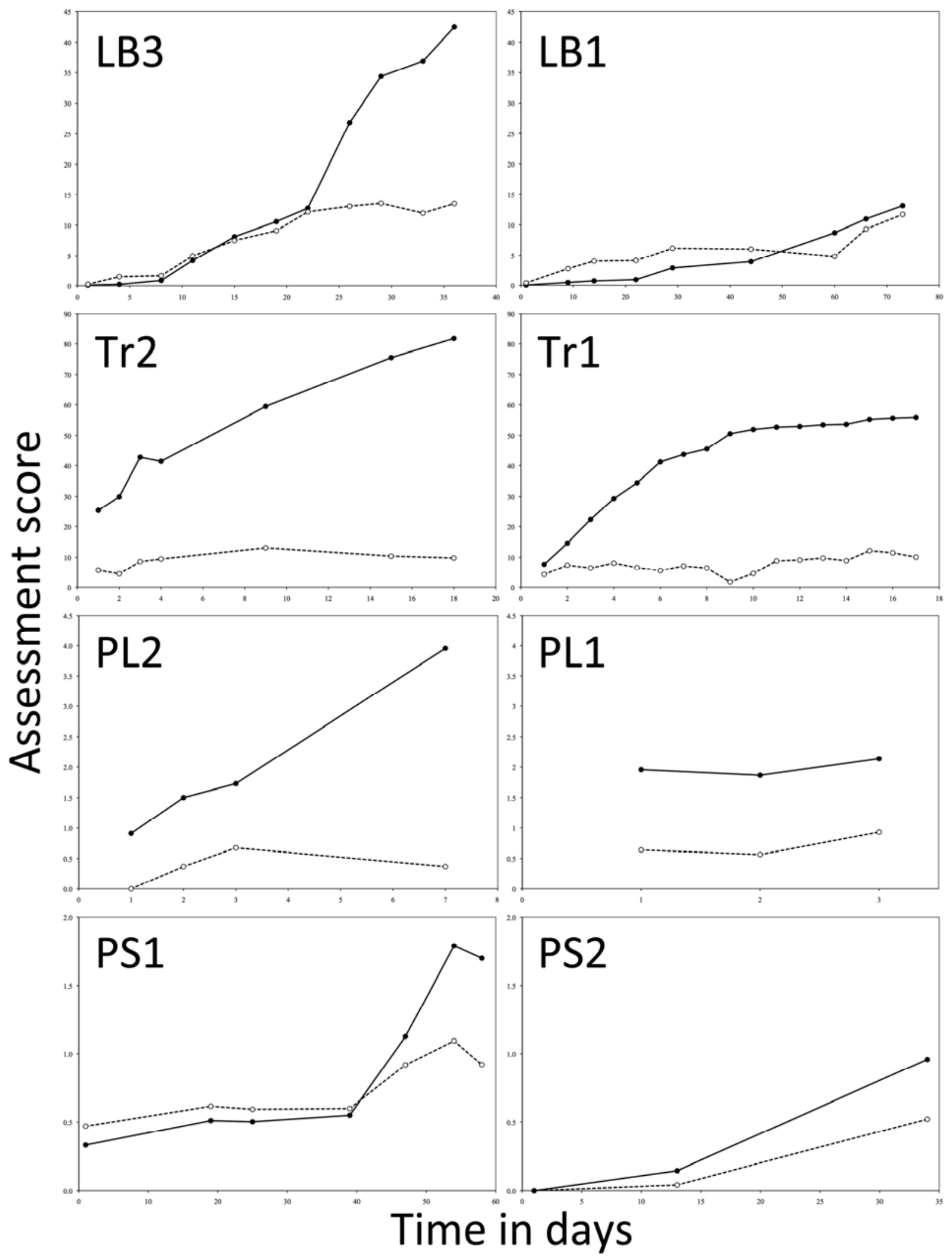

Fig. 3. Disease progress curves for three plant-pathogen and one plant-fungicide systems: $L B=$ late blight of potato, $\operatorname{Tr}=$ triforine effect on lettuce, $P L=$ powdery mildew of lettuce, and PS = powdery mildew of sugar beet. The trials shown in the left column have root of mean square error (RMSE) for standardized area under the disease progress curve (sAUDPC) higher than for standardized area under the disease progress stairs (sAUDPS), while the trials shown in the right column have RMSE for sAUDPC smaller than for sAUDPS. Full line indicates mean values of assessments, and dashed line shows RMSE for the assessments in each observation. For simplicity and uniformity of graphs, the $x$ axis for each trial starts at day 1, though the actual evaluations started at different days. 
pends on the standard deviation of individual observations $\left(\sigma_{i}\right)$. If the average standard deviation of the first and last observation is smaller than the average standard deviation of all other central observations

$$
\left(\bar{\sigma}_{1, n}<\bar{\sigma}_{c}, \text { where } \bar{\sigma}_{1, n}=\frac{\sigma_{1}+\sigma_{n}}{2} \text { and } \bar{\sigma}_{c}=\frac{\sum_{i=2}^{n-1} \sigma_{i}}{n-2}\right)
$$

then sAUDPS will outperform sAUDPC (Appendix C). Of course this limiting case itself will not occur in real data, but it is relevant when correlations are high and observations are performed regularly (or almost regularly). To test how this theoretical prediction works on real data, we compared $\bar{\sigma}_{1, n}$ and $\bar{\sigma}_{c}$ from the 50 analyzed trials. In 44 trials a prediction based on comparison of $\bar{\sigma}_{1, n}$ and $\bar{\sigma}_{c}$ matched RMSE values. Though these analyzed trials do not strictly meet both assumptions (perfect correlation and equal intervals), results correspond well with those obtained by comparing RMSE of sAUDPC and sAUDPS. Thus it is possible to predict a relative performance of the two formulas by comparing $\sigma_{i}$ values from individual observations. However, if assumptions of the perfect correlation and equal distribution of observations are not met, accuracy of predictions decreases.

Differences in values calculated by the AUDPC and AUDPS formulas. Because only half the weight is given to the first and last observation compared to intermediate observations in the AUDPC formula, this can lead to results that are not intuitively expected. Figure 4 shows assessment scores for two accessions. Accession A has a somewhat higher score than accession B in observations 2 to 5 , a somewhat smaller score in the first observation, but a substantially smaller score for the last observation. AUDPC for accession A is 14, while for accession B it is 13.5. On the contrary, the AUDPS score for accession A is smaller than for accession B (16 versus 19, respectively), the result that would be expected from the disease progress curve. In the special case, when assessments for all tested individuals in a trial have the same value for the first and last observations (e.g., all starting at $0 \%$, and ending at 100\%), a difference between AUDPS and AUDPC will be constant for all individuals and equals

$$
\frac{y_{1}+y_{n}}{2} \times \frac{D}{n-1}
$$

There is also a distinction between the AUDPC and AUDPS in the value of standardized scores. When observations are performed at regular intervals, sAUDPS is equal to the arithmetic mean of all assessments, whereas sAUDPC values are affected by a smaller weight given to the first and last observation. For example, if values of 2, 2, and 5 are assessed on days 1, 2, and 3, the sAUDPC score is 2.75 . The sAUDPS score is 3 , the same as the arithmetic mean of the three assessments (and in the same units).

Impact of disease duration on the AUDPC and AUDPS values. Van der Plank (chapter 12 in citation 13) suggested two hypotheses when describing the relationship between loss of yield and AUDPC. On these hypotheses, injury is proportional to the AUDPC.

"First, injury is proportional to the amount of disease. Two pustules do twice as much damage as one pustule, other things being equal."

"Second, injury is proportional to the duration of the disease. A pustule developed two weeks before the crop ripens does twice as much damage as a pustule developed one week before, other things being equal."

Both AUDPC and AUDPS give equivalent results regarding the first hypothesis; the values of AUDPC and AUDPS are always proportional to the disease score. For example, if accession A has disease scores 1, 2, and 5 in three consecutive days, and accession $\mathrm{B}$ has the disease scores of 2, 4, and 10 (double that of the acces- sion A scores), the AUDPC for the accession B is twice as high as for the accession A (10 versus 5). Similarly, AUDPS score for the accession B is twice the AUDPS score for the accession A (16 versus 8). However, in some cases there may be a difference between the two formulas when calculating values related to the second hypothesis. For example, if a disease is evaluated on accessions $\mathrm{C}, \mathrm{D}$, and $\mathrm{E}$ on four consecutive days and the accession $\mathrm{C}$ shows a disease only on the last observation (recorded as $0,0,0,1)$, the accession $\mathrm{D}$ shows the same disease score a day earlier $(0,0,1,1)$, and the accession $\mathrm{E}$ develops a disease already on the first day $(1,1,1,1)$. The AUDPC scores for the three accessions are $\mathrm{C}=0.5, \mathrm{D}=1.5, \mathrm{E}=3$. A disease on the accession $\mathrm{E}$ was observed as lasting twice as long as on the accession D (4 days versus 2 days), and the AUDPC score for the accession $\mathrm{E}$ is indeed twice as high (3 versus 1.5). But the ratio is not the same when the accessions $\mathrm{D}$ and $\mathrm{C}$ are compared. Though a disease on the accession D lasted twice as long as on the accession $\mathrm{C}$ (two days versus a single day), the AUDPC score for the accessions D is three times larger then for the accession $C$ ( 1.5 versus 0.5$)$. The respective AUDPS values for the three accessions are $\mathrm{C}=1, \mathrm{D}=$ 2 , and $E=4$, corresponding to the duration of a disease. Evaluation of the AUDPC and AUDPS formulas shows that though both of them have the same conceptual basis; their results are not always equivalent. When the evaluations are performed at regular intervals, AUDPS provides estimates that are always linearly proportional to the duration of a disease, while estimates based on AUDPC are not always linearly proportional to the duration of a disease.

Relative AUDPC and AUDPS. Relative AUDPC is calculated as a ratio between actual AUDPC and maximum potential AUDPC (2). The maximum potential AUDPC is the value that would be reached if assessments at every observation were at maximum $\left(y_{\max }\right)$. Similarly as for AUDPC, relative AUDPS (rAUDPS) is calculated as a ratio of actual and maximum potential AUDPS. However, because time span for AUDPS is different than for AUDPC, the rAUDPS formula is

$$
r A U D P S=\frac{A U D P S \times(n-1)}{D \times n \times y_{\max }}=\frac{s A U D P S}{y_{\max }}
$$

When a minimum potential assessment value is larger than zero $\left(y_{\min }>0\right)$, the rAUDPC and rAUDPS formulas need to be adjusted (e.g., for 1 to 9 rating scale). Theoretically, this adjustment should be carried out for any $y_{\min } \neq 0$, but in practice negative values are not used in disease progress assessments. The formula for relative AUDPS including adjustment for a nonzero minimum assessment is

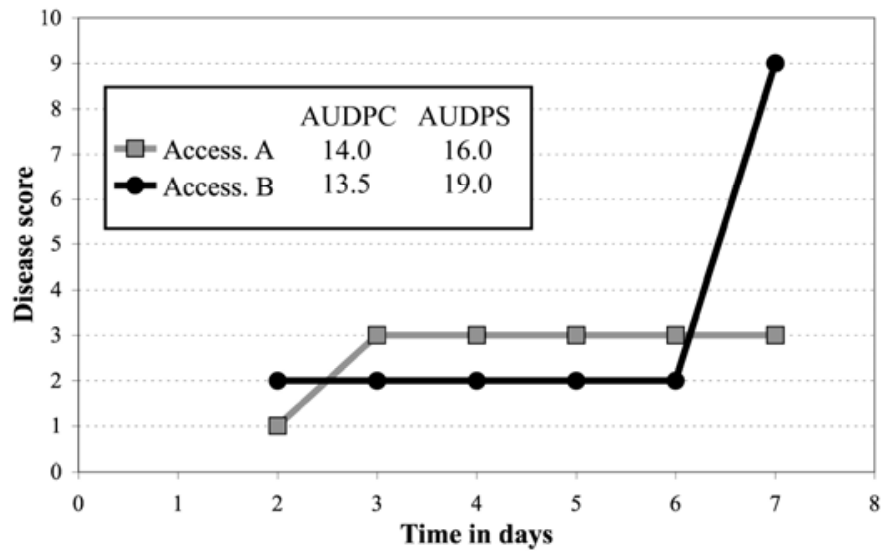

Fig. 4. Differences between area under the disease progress curve (AUDPC) and area under the disease progress stairs (AUDPS) scores for two hypothetical accessions. Accession A (gray line) has a higher AUDPC score, but lower AUDPS score than accession B (black line) because AUDPC formula gives only half a weight to the first and last observations. 


$$
r A U D P S=\frac{s A U D P S-y_{\min }}{y_{\max }-y_{\min }}
$$

In addition to relative AUDPC as described by Fry (2), other types of AUDPC were termed relative. These are based on a ratio of actual AUDPC, and AUDPC of reference accessions (14) or an accession with the highest AUDPC score in a trial (8). These statistics can also be calculated in the same way as rAUDPS values, but again, a proper adjustment is needed if $y_{\min }>0$.

Because relative AUDPC or AUDPS values are related to standardized sAUDPC and sAUDPS by a linear transformation, there seems little practical difference between standardized and relative versions of both measures. We preferred the standardized versions in the present paper because these allow for studying relative weights.

Conclusions. We developed a new formula to combine data from a disease progress curve into a single observation. AUDPS has the same conceptual basis and seeks to estimate the same quantity as AUDPC; it only uses a different estimation approach that is based on the slight extension of the limits of integration. This new formula gives a weight closer to optimal for the first and last observations that are undervalued by the AUDPC formula. Tests of precision on 50 trials indicate that sAUDPS outperforms sAUDPC in the trials where the average standard deviation of assessments from the first and last observation is smaller than the average standard deviation of assessments from all other (central) observations. We propose using this new formula for combining disease progress data assessed at multiple observations.

Since disease progress is frequently observed and data are recorded from the onset of disease until disease reaches a plateau or it is close to it, it is possible to assume that variance among replications within each accession in those early and late observations is smaller than the variance during midperiod of disease progress. If this assumption is correct and our formula for predicting relative performance of sAUDPC and sAUDPS applies, then sAUDPS outperforms sAUDPC frequently. Of course, if only a specific time period is studied that starts later than onset of a disease and/or finishes earlier than plateau of a disease, then sAUDPC might outperform sAUDPS. SAS codes for calculating AUDPS (equation 3) and sAUDPS (equation 7) are provided in Supplemental material 1.

\section{APPENDIX A}

To study the properties of the optimal weights, it is instructive to use the simple weights in equation 9 and assume for simplicity that all resulting weights are positive. Since $y$ values are usually repeated measures taken on the same plot, $V$ can entail a serial correlation structure. To gain some insight, we may consider the following special cases (assuming equally spaced data).

(i) Data are identically and independently distributed: $V=I \sigma^{2}$. The optimal weights are $w_{i}=n^{-1}$. These are the weights used in AUDPS, which is therefore optimal in this case.

(ii) Data have a compound symmetry structure $V=J \phi+I \sigma^{2}$, where $J=11^{T}$. Again, the optimal weights are $w_{i}=n^{-1}$.

(iii) $V$ is diagonal with elements $\sigma_{i}^{2}$, in other words, observations are independent, but variances differ between observations. The optimal weights are

$$
w_{i}=\sigma_{i}^{2}\left(\sum_{i=1}^{n} \sigma_{i}^{2}\right)^{-1}
$$

Thus, observations with large variance receive a smaller weight. In sAUDPC the first and last observation carry half the weight of intermediate observations. Thus, if the first or last observations have a comparatively large variance, sAUPDS may be less precise than sAUDPC. (iv) $V$ has an autoregressive first order structure $\operatorname{AR}(1)$ structure, i.e.,

$$
V=\sigma^{2}\left(\begin{array}{ccccc}
1 & \rho & \rho^{2} & \cdots & \rho^{n-1} \\
\rho & 1 & \rho & \cdots & \rho^{n-2} \\
\rho^{2} & \rho & 1 & \cdots & \rho^{n-3} \\
\vdots & \vdots & \vdots & \ddots & \vdots \\
\rho^{n-1} & \rho^{n-2} & \rho^{n-3} & \cdots & 1
\end{array}\right)
$$

where $0<\rho<1$ is the autocorrelation. Then,

$$
V^{-1}=\frac{1}{\sigma^{2}\left(1-\rho^{2}\right)}\left(\begin{array}{ccccc}
1+\rho^{2} & -\rho & 0 & \cdots & 0 \\
-\rho & 1+\rho^{2} & -\rho & \cdots & 0 \\
0 & -\rho & 1+\rho^{2} & \cdots & 0 \\
\vdots & \vdots & \vdots & \ddots & \vdots \\
0 & 0 & 0 & \cdots & 1+\rho^{2}
\end{array}\right)
$$

(page 160 in literature citation 7).

It follows that weights are

$$
w_{i}=\frac{1}{\sigma^{2}} \times\left(\frac{1}{1+\rho}+\frac{\rho}{1-\rho^{2}}\right)
$$

for the first and last observations and

$$
w_{i}=\frac{1}{\sigma^{2}} \times \frac{1}{1+\rho}
$$

for all other observations. Thus, the first and last observations have larger optimal weight than the other observations. In this situation, AUDPS is closer to the optimal weights than AUDPC, which has half the weight for the first and last observation compared with the other observations.

\section{APPENDIX B}

AUDPC and AUDPS use weights proportional to $v_{i}=\left(t_{i-1}+t_{i+1}\right) / 2$ for $i=2, \ldots, n-1$. Denote the factor of proportionality as $b$, such that $w_{i}=b v_{i}$ for $i=2, \ldots, n-1$. Now $b$ needs to be chosen such that the restriction

$$
\sum_{i=1}^{n} w_{i}=1
$$

is obeyed. Thus, we must have

$$
\sum_{i=1}^{n} w_{i}=w_{1}+b \sum_{i=2}^{n-2} v_{i}+w_{n}=1
$$

If we set

$$
c=b \sum_{i=2}^{n-2} v_{i}
$$

the constraint becomes $w_{1}+w_{n}+c=1$. The estimator can be written as

$$
L=w_{1} y_{1}+\sum_{i=2}^{n-1} w_{i} y_{i}+w_{n} y_{n}
$$

where the last term can be reexpressed as

$$
\sum_{i=2}^{n-1} w_{i} y_{i}=b \sum_{i=2}^{n-1} v_{i} y_{i}=\left(b \sum_{i=2}^{n-1} v_{i}\right) m=c m \text { with } m=\frac{\sum_{i=2}^{n-1} v_{i} y_{i}}{\sum_{i=2}^{n-1} v_{i}}
$$

Thus, the estimator can be written as $L=w_{1} y_{1}+c m+w_{n} y_{n}$ subject to the constraint $w_{1}+c+w_{n}=1$ (equation 10).

\section{APPENDIX C}

Let $V=\operatorname{var}(y)=S R S$, where $S$ is a diagonal matrix holding the standard deviations $\left(\sigma_{i}\right)$ of $y_{i}(i=1, \ldots, n)$ and $R$ is the correlation 
matrix. We consider the case of perfectly correlated observations, corresponding to $R=11^{T}$, where 1 is a vector of ones. In this case we find $\operatorname{var}(L)=w^{T} V w=\left(w^{T} S 1\right)^{2}$.

Thus, to evaluate precision of $L$, we only need to consider

$$
\sqrt{\operatorname{var}(L)}=w^{T} S 1=\bar{\sigma}_{\bullet}=\sum_{i=1}^{n} w_{i} \sigma_{i}
$$

the standard deviation of $L$. In other words, the standard deviation of $L$ is equal to the weighted mean of standard deviations of observations, with weights equal to those used in $L$ itself. Now assume equal spacing, meaning that $w_{1}=w_{n}=a$ and $w_{2}=w_{3}=\ldots=w_{n-1}=b$. For sAUDPS we have $a=b=n^{-1}$, while for sAUDPC $a=b / 2=[2(n-1)]^{-1}$. The standard deviation of $L$ can be expressed as $\sqrt{\operatorname{var}(L)}=2 a \bar{\sigma}_{1, n}+(n-2) b \bar{\sigma}_{c}$, Using the definitions of $a$ and $b$, we find that the standard deviation of sAUDPS is larger than that of sAUDPC if and only if

$$
\frac{2 \bar{\sigma}_{1, n}+(n-2) \bar{\sigma}_{c}}{n}>\frac{\bar{\sigma}_{1, n}+(n-2) \bar{\sigma}_{c}}{(n-1)}
$$

which after some algebraic rearrangement yields $\bar{\sigma}_{1, n}>\bar{\sigma}_{c}$. This confirms our proposition that the variance (standard deviation) of sAUDPS is larger than that of sAUDPC if and only if $\bar{\sigma}_{1, n}>\bar{\sigma}_{c}$ for the special case of equally spaced data and perfect positive correlation.

\section{ACKNOWLEDGMENTS}

We thank A. Atallah for technical assistance with field experiments and manuscript preparation, and L. V. Madden for critically reviewing the manuscript. R. Hayes, R. Lewellen, M. McGrath, and J. McCreight kindly provided the trial data.

\section{LITERATURE CITED}

1. Ewing, E. E., Simko, I., Smart, C. D., Bonierbale, M. W., Mizubuti, E. S. G., May, G. D., and Fry, W. E. 2000. Genetic mapping from field tests of qualitative and quantitative resistance to Phytophthora infestans in a population derived from Solanum tuberosum and Solanum berthaultii. Mol. Breed. 6:25-36.

2. Fry, W. E. 1978. Quantification of general resistance of potato cultivars and fungicide effects for integrated control of potato late blight. Phytopathology 68:1650-1655.

3. Haynes, K. G., and Weingartner, D. P. 2004. The use of area under the disease progress curve to assess resistance to late blight in potato germplasm. Am. J. Potato Res. 81:137-141.

4. Jeger, M. J., and Viljanen-Rollinson, S. L. H. 2001. The use of the area under the disease-progress curve (AUDPC) to assess quantitative disease resistance in crop cultivars. Theor. Appl. Genet. 102:32-40.

5. Madden, L. V., Hughes, G., and van den Bosch, F. 2007. The Study of Plant Disease Epidemics. American Phytopathological Society, St. Paul, MN.

6. Piepho, H. P., Büchse, A., and Richter, C. 2004. A mixed modelling approach to randomized experiments with repeated measures. J. Agron. Crop Sci. 190:230-247.

7. Rao, C. R., Toutenburg, Shalabh, H., and Heumann, C. 2008. Linear Models and Generalizations. 3rd extended ed. Springer, Berlin.

8. Rauscher, G., Simko, I., Mayton, H., Bonierbale, M., Smart, C. D., Grünwald, N. J., Greenland, A., and Fry, W. E. 2010. Quantitative resistance to late blight from Solanum berthaultii cosegregates with $R_{P i-b e r}$ : Insights in stability through isolates and environment. Theor. Appl. Genet. 121:1553-1567.

9. Simko, I. 2002. Comparative analysis of quantitative trait loci for foliage resistance to Phytophthora infestans in tuber-bearing Solanum species. Am. J. Potato Res. 79:125-132.

10. Simko I., Hayes, R. J., Truco, M. J., and Michelmore, R. W. 2011. Mapping a dominant negative mutation for triforine sensitivity in lettuce and its use as a selectable marker for detecting hybrids. Euphytica 182:157-166.

11. Simko, I., and Hu, J. 2008. Population structure in cultivated lettuce and its impact on association mapping. J. Am. Soc. Hort. Sci. 133:61-68.

12. Simko, I., Pechenick, D. A., McHale, L. K., Truco, M. J., Ochoa, O. E., Michelmore, R. W., and Scheffler, B. E. 2009. Association mapping and marker-assisted selection of the lettuce dieback resistance gene Tvrl. BMC Plant Biol. 9:135.

13. Van der Plank, J. E. 1963. Plant Diseases: Epidemics and Control. Academic Press, New York.

14. Yuen, J. E., and Forbes, G. A. 2009. Estimating the level of susceptibility to Phytophthora infestans in potato genotypes. Phytopathology 99:782786. 